\title{
The Muon Cooling RF R\&D Program
}

\author{
Y. Torun*, A. Bross ${ }^{\dagger}$, D. Li $^{* *}$, A. Moretti ${ }^{\dagger}$, J. Norem ${ }^{\dagger}$, Z. Qian ${ }^{\dagger}$, \\ R. A. Rimmer ${ }^{\S}$ and M. S. Zisman** \\ * Illinois Institute of Technology, Chicago, Illinois 60616, USA \\ ${ }^{\dagger}$ Fermi National Accelerator Laboratory, Batavia, Illinois 60510, USA \\ ${ }^{*}$ Lawrence Berkeley National Laboratory, Berkeley, California 94720, USA \\ ${ }^{\ddagger}$ Argonne National Laboratory, Argonne Illinois 60439, USA \\ ${ }^{\S}$ Jefferson Lab, Newport News, Virginia 23606, USA
}

\begin{abstract}
Cooling muon beams in flight requires absorbers to reduce the muon momentum, accelerating fields to replace the lost momentum in the longitudinal direction, and static solenoidal magnetic fields to focus the muon beams. The process is most efficient if both the magnetic fields and accelerating fields are high and the rf frequency is low. We have conducted tests to determine the operating envelope of high-gradient accelerating cavities in strong static magnetic fields. These studies have already produced useful information on dark currents, magnetic fields and breakdown in cavities. In addition to continuing our program at $805 \mathrm{MHz}$, we are starting to test a $201 \mathrm{MHz}$ cavity and are planning to look at a variety of appropriate geometries and materials. In parallel with these activities, we are supporting R\&D on models and surface structure.
\end{abstract}

PACS: 29.17.+w, 52.80.Vp, 73.22.2f

\section{IONIZATION COOLING}

Neutrino Factories and Muon Colliders hold the promise of neutrino and muon beams of unprecedented intensity, energy and quality and there is a strong worldwide effort on design studies and hardware R\&D toward the goal of building such facilities [1].

Ionization cooling [2] appears to be the only practical option for generating very high brightness muon beams [3] since the standard techniques (stochastic and electron cooling) are too slow for muons. Muons can be generated in large numbers only at low energies and therefore decay in a few microseconds. Transverse cooling involves simply passing the muon beam through an absorbing medium to shrink the momentum through ionization energy loss, and then restoring the longitudinal component of the momentum by rf acceleration, reducing the transverse emittance as a result. Efficient cooling using this method requires high-field solenoids for strong focusing, which rules out use of superconducting rf cavities.

The Neutrino Factory and Muon Collider Collaboration (NFMCC) [4] has been working to solve the technical challenges in Neutrino Factory and Muon Collider design [5]. Part of this effort is MuCool, the R\&D program at Fermilab for developing components for muon ionization cooling, including liquid-hydrogen absorbers [6, 7], solenoid magnets and rf cavities. The MuCool program also covers instrumentation, high-power beam tests and support of MICE [8] (the international Muon Ionization Cooling Experiment), an experiment using MuCool-developed components that is now under construction at RAL [9]. 


\section{MUCOOL RF R\&D}

The evolution of normalized transverse emittance, $\varepsilon$, of a muon beam as a function of distance $s$ in a solenoidal channel is given by [10]

$$
\frac{d \varepsilon}{d s} \simeq \frac{\left\langle\frac{d E}{d s}\right\rangle}{\beta^{2} E}\left(\varepsilon-\varepsilon_{0}\right)
$$

where $\beta$ and $E$ are the average muon speed and energy and $\varepsilon_{0}$, equilibrium emittance, is given by

$$
\varepsilon_{0} \simeq-\frac{0.875 \mathrm{MeV}}{\left\langle\frac{d E}{d s}\right\rangle X_{0}} \beta_{\perp} .
$$

The equilibrium emittance is directly proportional to the focusing length $\beta_{\perp}$, and inversely proportional to the energy loss per radiation length $X_{0}$. It is often referred to as the figure of merit for absorbers. In liquid hydrogen, which has the best figure of merit, energy loss is about $30 \mathrm{MeV} / \mathrm{m}$. To replace lost momentum in a hydrogen absorber, one needs rf cavities with large enough aperture for the muon beam. Since muon beams can go through thin windows unperturbed, $201 \mathrm{MHz}$ cavities with pillbox geometry have been proposed to make efficient use of rf power. They take up about four times the length of hydrogen for $16 \mathrm{MV} / \mathrm{m}$ peak accelerating gradient (which includes an extra factor of two for phase focusing) in the Feasibility Study II cooling channel [11] which is also the basis for MICE.

\section{MHz Program}

To gain experience with operation of rf cavities under these unusual conditions and to form a better understanding of the underlying physics and technical issues, initial tests were conducted at $805 \mathrm{MHz}$, in effect working with quarter-scale models, which were cheaper to build and easier to handle. The Lab-G facility at Fermilab was equipped with an rf system to operate a spare 805-MHz 12-MW klystron from the Fermilab Linac and a shielded cave for these tests. A superconducting magnet with $44 \mathrm{~cm}$ warm bore and two coils in a Helmholtz configuration was installed inside the cave. The coils can be powered individually, so it is possible to get various field distributions including a solenoidal mode for which the magnet is rated at $5 \mathrm{~T}$ as well as a gradient mode similar to that of the focus coils used in Study II and MICE.

First, a 1-m-long 805-MHz 6-cell open-iris cavity was installed in the magnet. This cavity had thin titanium windows at the ends which allowed dark current measurements on axis [12]. Dark currents from field emission appear to be precursors to damaging sparks in cavities [13], have a very steep dependence on gradient [14] and provide insight into the condition of the interior surfaces. A variety of diagnostics was used to characterize operating parameters and radiation around the cavity [15].

Next, a single-cell pillbox cavity with removable end plates was installed and used to map out the high-power conditioning behavior as a function of magnetic field. Although the cavity went up to $40 \mathrm{MV} / \mathrm{m}$ at zero field, the maximum stable operating gradient 
degraded quickly with the field turned on, dropping to about $35 \mathrm{MV} / \mathrm{m}$ at $0.25 \mathrm{~T}$ and 13.5 MV/m at $4 \mathrm{~T}[16]$.

Another approach to suppressing breakdown in cavities using high-pressure gas was also tested in Lab-G with an $805 \mathrm{MHz}$ pillbox prototype [17, 18]. Such pressurized cavities have the potential to provide ionization cooling for muon beams in addition to acceleration [19].

\section{Materials Research}

Reliable operation of cavities in high magnetic fields is essential for a Neutrino Factory cooling channel and high dark currents would be a potential problem for MICE [20]. An important part of the rf program is to identify practical materials, coatings and surface processing techniques that will allow cavities to withstand high gradients. Any deviations from a perfectly smooth surface on the interior of a cavity left over from the manufacturing process can lead to local enhancement of the electric field and increased dark current. This can generate concentrated heating and very large forces on the surface causing melting [21], mechanical break-up [12] and field evaporation of clusters [22, 23]. Sparks can cause pitting of the $\mathrm{Cu}$ surface and deposition of molten $\mathrm{Cu}$ over the interior and the resulting rough features on the surface can act as new sources of emission. Analysis of dark current data from MuCool cavities seems to indicate that the observed gradient limits are consistent with the tensile strength of $\mathrm{Cu}$. In addition, visual inspection of the cavities after operation in Lab-G has revealed useful hints on mitigating breakdown problems. When the $\mathrm{Cu}$ end plates that showed heavy pitting were replaced with TiN coated Be windows, the damage was confined to $\mathrm{Cu}$ surfaces. A special end flange has been built for the $805 \mathrm{MHz}$ pillbox cavity that can accommodate buttons of different materials. Using buttons with curvature so that the surface field is highest in that region, we will systematically explore the limits of different materials and coatings.

\section{MuCool Test Area}

A dedicated long-term test facility with rf power, cryogenics infrastructure and highpower beam is required to carry out MuCool $R \& D$ on rf cavities, liquid-hydrogen absorbers and instrumentation. The south end of the Fermilab Linac was a suitable location to build such a facility [24, 25]. Civil construction was completed in 2003 and the rf hardware has now been moved there (see Fig. 1). Both $12 \mathrm{MW}$ at $805 \mathrm{MHz}$ and 5 MW at $201 \mathrm{MHz}$ are available from the Linac. A beamline to bring the 400-MeV Linac $\mathrm{H}^{-}$beam into the area is designed and installation is expected in the near future.

\section{MHz Cavity}

The full-scale prototype cavity has a rounded cylindrical profile of about $1.2 \mathrm{~m}$ diameter and a $43 \mathrm{~cm}$ gap. It was built [26] by e-beam welding half-shells spun from 

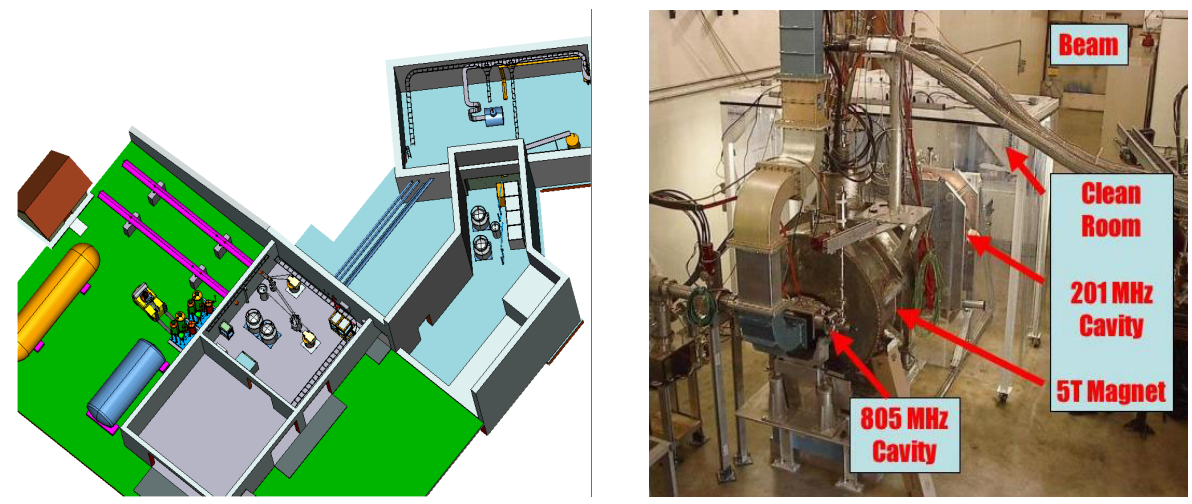

FIGURE 1. Layout of the MTA facility (left) and experimental hall (right)

6-mm-thick $\mathrm{Cu}$ sheet. Cooling tubes are welded to the outer surface and ports pulled on the equator for vacuum, rf power and diagnostic connections. Tuning and beam iris rings were also e-beam welded to each side. The interior was processed by mechanical polishing, light chemical cleaning and electropolishing for a clean and smooth finish to minimize field emission. In MICE, like in a Neutrino Factory cooling channel, the cavities would be in vacuum. Since this prototype is intended for testing in air, the cavity body is attached to thick aluminum plates to support it against atmospheric pressure. These plates also include attachment points for tuning rods to adjust the frequency through slight elastic deformation of the cavity shape and a frame to support its considerable weight. The cavity irises are terminated by $42-\mathrm{cm}$-diameter $0.38-\mathrm{mm}$ thick curved Be windows mounted with the concave/convex sides pointing in the same direction to minimize the frequency shift when they are distorted due to heating during high power operation. A detailed finite-element analysis has been performed [27] to establish the rf, thermal and structural behavior of the cavity. The design values [28] for the frequency, shunt impedance and quality factor are $201.25 \mathrm{MHz}, 22 \mathrm{M} \Omega / \mathrm{m}$ and 53500 , respectively, and the cavity should provide a gradient of $8 \mathrm{MV} / \mathrm{m}$ at $1 \mathrm{MW}$ and 16.2 MV/m at 4.6 MW input power. The cavity has been installed at the MTA and will be powered soon.

\section{STATUS}

The $805 \mathrm{MHz}$ cavity is ready for high-power testing in a magnetic field with a pair of curved Be windows to establish stable operation. A program of systematic tests using buttons made of different materials and coatings will follow, to compare their suitability and to benchmark models of rf breakdown. Installation of the $201 \mathrm{MHz}$ cavity should be complete soon and we intend to make the first measurements of radiation around the cavity at $8 \mathrm{MV} / \mathrm{m}$ to check previous estimates for MICE detector backgrounds. Successful conditioning and reliable operation of this full size prototype cavity is an important milestone in the $\mathrm{MuCool} \mathrm{rf}$ program. It is desirable to operate this cavity in a 
large magnetic field as well. However, we do not yet have a magnet with large enough bore available for this purpose (a prototype for the coupling coil [29] in the cooling channel will be built in the future as funds allow). Since the effect of the magnetic field turns on rapidly at fields as low as $0.1 \mathrm{~T}$ which is the level of fringe field from the existing solenoid at one end of the cavity, we expect some guidance from operating this cavity with the magnet turned on.

\section{ACKNOWLEDGMENTS}

This work was supported by the U. S. Department of Energy, Office of High Energy Physics and the Illinois Board of Higher Education.

\section{REFERENCES}

1. Y. Torun, H. Haseroth, S. Machida and T. Yokoi, Nucl. Phys. B (Proc. Suppl.) 149, p. 251 (2005).

2. G. I. Budker and A. N. Skrinsky, Sov. Phys. Usp. 21, 277 (1978).

3. D. Neuffer, Particle Accelerators 14, 75 (1983).

4. http://www.cap.bnl.gov/mumu/

5. M. M. Alsharoa et al., Phys. Rev. ST Accel. Beams 6, 081001 (2003).

6. M. A. Cummings, these proceedings.

7. C. Darve, B. Norris and L. Pei, these proceedings.

8. http://mice.iit.edu/

9. D. M. Kaplan, these proceedings.

10. D. Neuffer, in Advanced Accelerator Concepts, ed. F. E. Mills, AIP Conf. Proc. 156 (AIP, New York, 1987), p. 201; R. C. Fernow and J. C. Gallardo, Phys. Rev. E 52, 1039 (1995).

11. Feasibility Study-II of a Muon-Based Neutrino Source, ed. S. Ozaki et al., BNL Report 52623 (2001).

12. J. Norem et al., Phys. Rev. ST Accel. Beams 6, 072001 (2003).

13. N. S. Xu and R. V. Latham, J. Phys. D: Appl. Phys. 27, 2547 (1994).

14. R. Fowler and L. Nordheim, Proc. R. Soc. A 119, 173 (1928).

15. P. Gruber and Y. Torun, Proceedings of the 2003 Particle Accelerator Conference (PAC03), Portland, OR, 2003 (IEEE, New York, 2003), p. 1413.

16. A. Moretti et al., Phys. Rev. ST Accel. Beams 8, 072001 (2005).

17. P. M. Hanlet et al., Proceedings of the 2005 Particle Accelerator Conference, Knoxville, TN, 2005.

18. K. Yonehara et al., Nucl. Phys. B (Proc. Suppl.) 149, pp. 286-288 (2005).

19. R. Johnson et al., these proceedings.

20. R. Sandström, Nucl. Phys. B (Proc. Suppl.) 149, pp. 301-302 (2005).

21. Proceedings of the 2003 Particle Accelerator Conference (PAC03), Portland, OR, 2003 (IEEE, New York, 2003), p. 1284.

22. J. Norem, Z. Insepov and I. Konkashbiev, Nucl. Instr. and Meth. In Phys. Res. A 537, 510 (2005).

23. Z. Insepov, J. Norem and A. Hassanein, Phys. Rev. ST Accel. Beams 7, 122001 (2004).

24. M. Popovic, Proceedings of the 2004 European Particle Accelerator Conference (EPAC2004), Lucerne, Switzerland, 2004.

25. C. Johnstone, A. Bross and I. Rakhno, Proceedings of the 2005 Particle Accelerator Conference, Knoxville, TN, 2005.

26. R. A. Rimmer et al., ibid.

27. S. Virostek and D. Li, ibid.

28. D. Li et al., Proceedings of the 2003 Particle Accelerator Conference (PAC03), Portland, OR, 2003 (IEEE, New York, 2003), p. 1243.

29. M. A. Green et al., Proceedings of the 2005 Particle Accelerator Conference, Knoxville, TN, 2005. 